\title{
Evidence for the importance of angiotensin II type 1 receptor in ischemia-induced angiogenesis
}

\author{
Ken-ichiro Sasaki, ${ }^{1}$ Toyoaki Murohara, ${ }^{1}$ Hisao Ikeda, ${ }^{1}$ Takeshi Sugaya, ${ }^{2}$ \\ Toshifumi Shimada, ${ }^{1}$ Satoshi Shintani, ${ }^{1}$ and Tsutomu Imaizumi ${ }^{1}$ \\ ${ }^{1}$ The Cardiovascular Research Institute, and Department of Internal Medicine III, Kurume University School of Medicine, \\ Kurume, Japan \\ ${ }^{2}$ Discovery Research Laboratory, Tanabe Seiyaku Co., Kashima, Osaka, Japan
}

Address correspondence to: Toyoaki Murohara, The Cardiovascular Research Institute, Kurume University School of Medicine, 67 Asahi-machi, Kurume, 830-0011 Japan.

Phone: 81-942-35-3311 ext. 3801; Fax: 81-942-31-7707; E-mail: toyom@med.kurume-u.ac.jp.

This study was presented in part at the Scientific Sessions 2001 of the American Heart Association Conference, Anaheim, California, USA, on November 13, 2001.

Received for publication April 16, 2001, and accepted in revised form December 27, 2001.

The role of the renin-angiotensin system (RAS) in angiogenesis is little known. Here, we show that the angiotensin II (ATII) type 1 (AT1) receptor plays an important role in ischemia-induced angiogenesis. Well-developed collateral vessels and angiogenesis were observed in wild-type (WT) mice in response to hindlimb ischemia, whereas these responses were reduced in ATII type 1a receptor knockout $\left(A T 1 a^{-/}\right)$mice. Ischemia-induced angiogenesis was also impaired in WT mice treated with the AT1 receptor blocker TCV-116. These effects were not due to reduced systemic blood pressure (SBP), because hydralazine treatment preserved angiogenesis in WT mice although it reduced SBP to a level similar to that of $A T 1 a^{-/-}$mice. Infiltration of inflammatory mononuclear cells (MNCs), including macrophages and $\mathrm{T}$ lymphocytes, was suppressed in the ischemic tissues of $A T 1 a^{-/-}$mice compared with WT mice. Double immunofluorescence staining revealed that infiltrated macrophages and T lymphocytes expressed VEGF, and the expression of VEGF and monocyte chemoattractant protein-1 was also decreased in $A T 1 a^{-/}$. Finally, the impaired angiogenesis in $A T 1 a^{-/-}$mice was rescued by intramuscular transplantation of MNCs obtained from WT mice, further indicating the importance of MNC infiltration in ischemia-induced angiogenesis. Thus, the ATII-AT1 receptor pathway promotes early angiogenesis by supporting inflammatory cell infiltration and angiogenic cytokine expression.

$$
\text { J. Clin. Invest. 109:603-611 (2002). DOI:10.1172/JCI200213055. }
$$

\section{Introduction}

The renin-angiotensin system (RAS) plays essential roles in the maintenance of vascular homeostasis (1). Recent large-scale clinical trials demonstrated that angiotensinconverting enzyme (ACE) inhibitors reduced not only the mortality rate due to cardiovascular disease but also the rate of tumor-related death (2). Because tumor growth depends on angiogenesis $(3,4)$, ACE inhibitors might inhibit angiogenesis and thereby retard tumor growth. However, there are several controversial reports regarding the effects of ACE inhibitors on angiogenesis. For example, the ACE inhibitor lisinopril is reported to suppress diabetic retinopathy, which is characterized by pathological angiogenesis on the retina (5). Likewise, Volpert et al. (6) showed that captopril suppresses tumor angiogenesis and growth. In contrast, Fabre et al. (7) reported that quinapril augments angiogenesis in a rabbit model of hindlimb ischemia, leaving the precise role of the RAS in angiogenesis unclear. Furthermore, in these previous studies, ACE inhibitors were used to suppress the functions of the RAS. However, there are limitations regarding the use of ACE inhibitors to examine the role of the RAS in vivo. First, ACE inhibitors suppress not only angiotensin II (ATII) syn- thesis but also the breakdown of bradykinin (8). This would stimulate endothelial nitric oxide release (8), which in turn might promote angiogenesis $(7,9,10)$. Second, because ATII is produced not only by ACE, but also by other enzymes, such as chymase (11), it is not possible to evaluate the role of ATII in angiogenesis in vivo using ACE inhibitors alone.

There are two major subtypes of ATII receptors, AT1 and AT2. In mice, the AT1 receptor is further subdivided into AT1a and AT1b receptors (12). Most of the well-known ATII functions in the cardiovascular system are mediated through the AT1 receptor $(13,14)$, especially through the AT1a receptor in rodents (15). We and other investigators recently generated AT1a knockout $\left(A T 1 a^{-/}\right)$mice that showed reduced systemic blood pressure (SBP) $(16,17)$. Accordingly, we used these mice to examine the role of the AT1a receptor in angiogenesis, employing a well-established mouse model of ischemia-induced angiogenesis.

\section{Methods}

Animals. To obtain AT1a-deficient heterozygous $\left(A T 1 a^{+/-}\right)$ mice that have $\mathrm{C} 57 \mathrm{BL} / 6$ background, a germline chimera derived from TT2 embryonic stem cells with a targeted 
mutation of the AT1a gene as described previously (16) was backcrossed for five generations with C57BL/6 mice (18). The resulting $A T 1 a^{+/} \mathrm{F}_{5}$ mice were then intercrossed to generate the homozygous $\left(A T 1 a^{-/}\right)$mice $(16,18)$. The $A T 1 a^{-/-}$mice were then inbred to obtain an appropriate number of animals for the present study. As wild-type $\left(\mathrm{WT} ; A T 1 \mathrm{a}^{++}\right)$mice, C57BL/6-strain mice were obtained from Clea Japan Inc. (Tokyo, Japan). Male mice at the age of 8-10 weeks were used.

Mouse model of angiogenesis. The study protocols were approved by the Institutional Animal Care and Use Committee of Kurume University School of Medicine. We used a mouse model of angiogenesis, in which the entire left femoral artery and vein were excised surgically $(10,19)$. When hindlimb ischemia was induced, new blood vessels grew into the ischemic limb $(10,19)$. We prepared this model in $A T 1 a^{-/}$mice and WT mice and employed various pharmacological agents to determine whether ischemia-induced angiogenesis was affected by the deficiency of the AT1a receptor. In brief, mice were subjected to unilateral hindlimb ischemia under anesthesia with sodium pentobarbital $(50 \mathrm{mg} / \mathrm{kg}$ intraperitoneally) (10). Before surgery and on postoperative days $3,7,14,21,28$, and 35 , body weight and SBP were determined. SBP was determined using a tail-cuff pressure analysis system (TK370C; UNICOM, Tokyo, Japan) in the conscious state. Capillary angiogenesis, collateral vessel formation (arteriogenesis), and limb blood flow were examined by the following methods.

Laser Doppler blood flow analysis. We measured hindlimb blood flow using a laser Doppler blood flow (LDBF) analyzer (Moor LDI; Moor Instruments, Devon, United Kingdom) as described previously (10). Before and on postoperative days 3, 7, 14, 21, 28, and 35 , we performed LDBF analysis over the legs and feet. Blood flow (i.e., blood cell movement) was displayed as changes in the laser frequency using different color pixels. After scanning, the stored images were analyzed to quantify blood flow, and mean LDBF values of the ischemic and nonischemic legs were calculated. To avoid data variations due to ambient light and temperature, hindlimb blood flow was expressed as the ratio of left (ischemic) to right (nonischemic) LDBF.

Angiographic score. On postoperative day 14, under pentobarbital anesthesia $(50 \mathrm{mg} / \mathrm{kg}$ intraperitoneally), the peritoneal cavity was opened, and a 26-gauge softtip catheter was inserted through the abdominal aorta. The lower limbs were gently perfused with $0.5 \mathrm{ml}$ of warm saline containing heparin $(10 \mathrm{U} / \mathrm{ml})$. Postmortem angiography was then performed by injecting $0.3 \mathrm{ml}$ of contrast media through the catheter at a perfusion pressure of $80-90 \mathrm{mmHg}$. X-ray angiograms were taken using a mammography system (Senographe 500T; GE Medical Systems, Paris, France), and the extent of collateral vessel formation was quantified by angiographic score as described previously (20).

Capillary density. Capillary density within the ischemic thigh adductor skeletal muscles was analyzed to obtain specific evidence of vascularity at the level of microcir- culation. Three pieces of ischemic muscles were harvested from each animal, sliced, and fixed in methanol. Tissues were embedded in paraffin, and multiple tissue slices, $5 \mu \mathrm{m}$ in thickness, were prepared. Capillary endothelial cells (ECs) were identified by immunohistochemical staining with a rat anti-mouse CD31 mAb (PharMingen, San Diego, California, USA). Fifteen random microscopic fields from three different sections in each tissue block were examined for the presence of capillary ECs, and capillary density was expressed as the number of capillaries per high power field $(\times 400)$.

Effects of hydralazine on ischemia-induced angiogenesis in WT mice. AT1 $a^{-1-}$ mice had lower SBP than WT mice $(16,17)$. We thus examined whether the lower SBP itself influenced ischemia-induced angiogenesis. We examined the effects of the reduction of SBP by hydralazine (Novartis Pharmaceuticals Corp., Tokyo, Japan) on angiogenesis in nine WT mice. SBP was reduced by oral hydralazine $(50 \mathrm{mg} / \mathrm{kg} / \mathrm{d})$ in WT mice; our preliminary experiments showed that the SBP in WT mice treated with this dose of hydralazine was similar to that of $A T 1 a^{-/}$mice. On day 10 of hydralazine treatment, hindlimb ischemia was induced, and the ischemic/normal LDBF ratio was examined up to 35 days after surgery.

Effects of TCV-116 on ischemia-induced angiogenesis in WT mice. We examined whether selective pharmacological blockade of the AT1 receptor by candesartan cilexetil (TCV-116; Takeda Chemical Industries, Osaka, Japan) $(12,13,21)$ in WT mice mimicked the altered angiogenic response observed in $A T 1 a^{-/-}$mice. TCV-116 is a specific AT1 receptor antagonist, and its AT1-binding affinity is 80 times greater than that of losartan and 10 times greater than that of EXP 3174, the active form of losartan $(12,13)$. TCV-116 has been used to block the major vascular effects of ATII in previous in vivo studies (21-23). In preliminary experiments, we examined the doseresponse effects of oral TCV-116 on SBP in WT mice. We used $5 \mathrm{mg} / \mathrm{kg} / \mathrm{d}$ of TCV-116, because this dose reduced SBP in WT mice to a level similar to that of $A T 1 a^{-/-}$mice. TCV-116 was administered via drinking water to $15 \mathrm{WT}$ mice. On day 10 of the administration, mice were subjected to hindlimb ischemia, and ischemic/normal LDBF ratios were examined up to 35 days after surgery.

Effects of PD123319 on ischemia-induced angiogenesis in $A T 1 a^{-/-}$mice. To further test the biological role of ATII in the ischemia-induced angiogenesis, we examined the effects of PD123319, a selective AT2 receptor antagonist, on angiogenesis in $A T 1 a^{-/-}$mice with hindlimb ischemia. PD123319 has been used to block the biological effects of the ATII-AT2 receptor pathway in various in vivo experiments. We chose the dose of PD123319 $(30 \mathrm{mg} / \mathrm{kg} / \mathrm{d})$ that effectively suppressed AT2 receptor function in previous studies (24). PD123319 was administered using an osmotic pump (ALZA Corp., Palo Alto, California, USA) to five $A T 1 a^{-/-}$mice, which were then subjected to surgical hindlimb ischemia. Ischemic/normal LDBF ratios were examined up to 35 days after surgery. 
Histological analysis of the inflammatory responses. ATII has been shown to play a proinflammatory role (25-27). We thus examined whether the extent of inflammatory reactions in the ischemic tissues differed between WT and $A T 1 a^{-/-}$mice. Five-micrometer-thick multiple sections prepared from paraffin-embedded tissues of the ischemic limbs were used for histological analysis. Leukocyte infiltration was examined by hematoxylin and eosin (H\&E) staining as well as immunohistochemical staining using an anti-mouse CD45 $\mathrm{mAb}$ (PharMingen), a common leukocyte antigen.

VEGF expression in inflammatory mononuclear cells. Inflammatory mononuclear cells (MNCs), mainly macrophages and $\mathrm{T}$ lymphocytes, release various angiogenic cytokines including $\operatorname{VEGF}(19,28)$. Therefore, we also examined VEGF expression in MNCs in ischemic tissues using a double immunofluorescence staining technique. Cryostat sections, $5 \mu \mathrm{m}$ in thickness, from ischemic tissues were mounted on silicone-coated slides. They were then incubated overnight at $4^{\circ} \mathrm{C}$ with an anti-mouse VEGF mAb (Santa Cruz Biotechnology Inc., Santa Cruz, California, USA), and with either rat anti-mouse $\mathrm{F} 4 / 80 \mathrm{mAb}$ or rat anti-mouse $\mathrm{CD} 3 \mathrm{mAb}$ (Serotec Inc., Raleigh, North Carolina, USA) in a moist chamber. The slides were then incubated for 30 minutes at $37^{\circ} \mathrm{C}$ with an FITC-conjugated anti-goat IgG secondary antibody (Zymed Laboratories Inc., South San Francisco, California, USA) to detect VEGF. Then they were further incubated for 30 minutes at $37^{\circ} \mathrm{C}$ with TRITC-conjugated anti-rat IgG (Jackson ImmunoResearch Laboratories Inc., West Grove, Pennsylvania, USA) to detect macrophages or $\mathrm{T}$ lymphocytes. The slides were examined and photographed using a laser confocal microscope (Noran Instruments Inc., Middleton, Wisconsin, USA). The infiltrated macrophages, $\mathrm{T}$ lymphocytes, and VEGF-positive leukocytes per high power field $(\times 400)$ were then counted.

Western blot analysis. Protein extracts were obtained from homogenized ischemic skeletal muscles. One hundred micrograms of protein per sample was separated on a $12.5 \%$ polyacrylamide gel and electroblotted onto PVDF membranes (Trans-blot; Bio-Rad Laboratories Inc., Hercules, California, USA). The membrane was blocked with $10 \%$ nonfat dry milk in PBS with $0.2 \%$ Tween (T-PBS) and then probed with 1:100 of a goat polyclonal anti-mouse VEGF antibody (clone P-20) or with 1:100 of a goat polyclonal anti-mouse monocyte chemoattractant protein-1 (MCP-1) (clone M-18; Santa Cruz Biotechnology Inc.) for 3 hours at room temperature. After incubation with the primary antibody, the membrane was washed three times in T-PBS and then incubated for 1 hour with 1:5000 of anti-goat IgG conjugated with horseradish peroxidase (Santa Cruz Biotechnology Inc.). The membrane was then washed in T-PBS, and antigen-antibody complexes were visualized using an enhanced chemiluminescence kit (ECL; Amersham Biosciences, Buckinghamshire, United Kingdom) at room temperature, followed by exposure to x-ray films (Hyperfilm; Amersham Biosciences).
Isolation of peripheral blood MNCs from WT or AT1 $a^{-/-}$ mice and implantation into the ischemic hindlimb of $\mathrm{AT} \mathrm{a}^{-/-}$ mice. Peripheral blood was obtained from WT and $A T 1 a^{-/-}$mice. MNCs were isolated by centrifugation through a Histopaque-1083 density gradient (Sigma Chemical Co., St. Louis, Missouri, USA). In $14 A T 1 a^{-/-}$ mice, left hindlimb ischemia was induced, and WT mouse-derived MNCs $\left(4 \times 10^{5}\right.$ cells per animal, $\left.n=8\right)$ or $A T 1 a^{-/-}$mouse-derived MNCs $\left(4 \times 10^{5}\right.$ cells per animal, $n=6$ ) were implanted into the thigh adductor muscles in the ischemic limb on postoperative day 3 . In five additional mice, neutralizing anti-mouse VEGF mAb (R\&D Systems Inc., Minneapolis, Minnesota, USA) was continuously administered by a subcutaneously implanted osmotic pump (ALZA Corp.), and ischemia-induced angiogenesis was examined in $A T 1 a^{-/-}$mice that had been subjected to WT-derived MNC transplantation. The ischemic/normal LDBF ratio was examined up to 35 days after surgery.

Peritonitis model for in vivo leukocyte transendothelial migration assay. To examine the effects of the AT1a receptor deficiency on in vivo leukocyte transendothelial migration, we employed an additional inflammation model: a murine peritonitis model induced by injecting oyster glycogen (intraperitoneally). This is an established model used to examine the degree of leukocyte transendothelial migration in response to inflammation in vivo $(29,30)$. $A T 1 a^{-/-}(n=10)$ and WT mice $(n=10)$ were lightly anesthetized with pentobarbital ( $30 \mathrm{mg} / \mathrm{kg}$ intramuscularly), and $2 \mathrm{ml}$ of $0.1 \mathrm{vol} \%$ oyster glycogen type II (Sigma Chemical Co.) in sterile saline was injected into the peritoneal cavity. Four hours after the injection, the abdominal wall was cut and the peritoneal cavity was opened with special care to avoid blood contamination. The peritoneal cavity was washed with $2 \mathrm{ml}$ of warm heparinized $(10 \mathrm{U} / \mathrm{ml})$ saline; then peritoneal fluid was collected and centrifuged at $400 \mathrm{~g}$ for 10 minutes. The pellets were resuspended in PBS $(1 \mathrm{ml})$, and the total number of transmigrated leukocytes was calculated using a hemocytometer (K-800; Sysmex, Kobe, Japan).

Statistics. All values are presented as the mean \pm SE. All data were subjected to ANOVA followed by Fisher's analysis for comparison between any two means. Probabilities of less than 0.05 were considered to be statistically significant.

\section{Results}

Unilateral hindlimb ischemia. All mice survived after surgical induction of unilateral hindlimb ischemia and looked healthy during the follow-up period. Body weight did not differ among the groups throughout the experimental period (Table 1). Immediately after the left femoral artery and vein were resected, the ratio of ischemic (left) to normal (right) hindlimb blood flow decreased from 1.0 to $0.12 \pm 0.01$ in WT and $0.12 \pm 0.02$ in $A T 1 a^{-/-}$mice $(P=$ not significant [NS]). Thus, the severity of induced ischemia was comparable in the two groups. 
Table 1

Blood pressure and body weight of mice in each experimental group

\begin{tabular}{|c|c|c|c|c|c|c|c|}
\hline $\mathrm{SBP}(\mathrm{mmHg})(n)$ & Before & Day 3 & Day 7 & Day 14 & Day 21 & Day 28 & Day 35 \\
\hline $\begin{array}{l}\text { WT }(10) \\
\text { AT1 } a^{-/-}(9) \\
\text { WT + TCV-116 (8) } \\
\text { WT + Hyd (9) } \\
\text { AT1 } a^{-/-}+\text {PD123319 (5) } \\
\text { AT1 } a^{-/-}+\text {WT-MNCs }(8) \\
\text { AT1 } a^{-/-}+\text {AT1 } a^{-/-} \text {MNCs (6) } \\
\text { AT1 } a^{-/-}+\text {WT-MNCs + VEGF mAb (5) }\end{array}$ & $\begin{array}{l}94 \pm 2 \\
72 \pm 4^{\mathrm{A}} \\
70 \pm 2^{\mathrm{A}} \\
70 \pm 2^{\mathrm{A}} \\
82 \pm 2^{\mathrm{A}} \\
72 \pm 1^{\mathrm{A}} \\
73 \pm 2^{\mathrm{A}} \\
74 \pm 1^{\mathrm{A}}\end{array}$ & $\begin{array}{l}95 \pm 1 \\
71 \pm 4^{\mathrm{A}} \\
68 \pm 2^{\mathrm{A}} \\
71 \pm 1^{\mathrm{A}} \\
\mathrm{NM} \\
\mathrm{NM} \\
\mathrm{NM} \\
\mathrm{NM}\end{array}$ & $\begin{array}{l}96 \pm 2 \\
73 \pm 4^{\mathrm{A}} \\
69 \pm 2^{\mathrm{A}} \\
70 \pm 2^{\mathrm{A}} \\
79 \pm 3^{\mathrm{A}} \\
74 \pm 2^{\mathrm{A}} \\
75 \pm 2^{\mathrm{A}} \\
78 \pm 1^{\mathrm{A}}\end{array}$ & $\begin{array}{l}98 \pm 2 \\
73 \pm 3^{\mathrm{A}} \\
72 \pm 1^{\mathrm{A}} \\
70 \pm 1^{\mathrm{A}} \\
79 \pm 2^{\mathrm{A}} \\
75 \pm 1^{\mathrm{A}} \\
74 \pm 2^{\mathrm{A}} \\
76 \pm 1^{\mathrm{A}}\end{array}$ & $\begin{array}{l}98 \pm 2 \\
73 \pm 4^{\mathrm{A}} \\
68 \pm 1^{\mathrm{A}} \\
71 \pm 1^{\mathrm{A}} \\
82 \pm 3^{\mathrm{A}} \\
76 \pm 0^{\mathrm{A}} \\
78 \pm 2^{\mathrm{A}} \\
76 \pm 1^{\mathrm{A}}\end{array}$ & $\begin{array}{l}98 \pm 3 \\
74 \pm 4^{\mathrm{A}} \\
69 \pm 1^{\mathrm{A}} \\
72 \pm 1^{\mathrm{A}} \\
83 \pm 2^{\mathrm{A}} \\
74 \pm 1^{\mathrm{A}} \\
79 \pm 3^{\mathrm{A}} \\
75 \pm 1^{\mathrm{A}}\end{array}$ & $\begin{array}{l}99 \pm 2 \\
74 \pm 4^{\mathrm{A}} \\
69 \pm 1^{\mathrm{A}} \\
71 \pm 1^{\mathrm{A}} \\
84 \pm 2^{\mathrm{A}} \\
76 \pm 2^{\mathrm{A}} \\
77 \pm 1^{\mathrm{A}} \\
76 \pm 1^{\mathrm{A}}\end{array}$ \\
\hline Body weight $(\mathrm{g})(n)$ & Before & Day 3 & Day 7 & Day 14 & Day 21 & Day 28 & Day 35 \\
\hline $\begin{array}{l}\text { WT }(10) \\
\text { AT1 } a^{-/-}(9) \\
\text { WT + TCV-116 (8) } \\
\text { WT + Hyd (9) } \\
\text { AT1 } a^{-/-}+\text {PD123319 (5) } \\
\text { AT1 } a^{-/-}+\text {WT-MNCs }(8) \\
\text { AT1 } a^{-/-}+\text {AT1 } a^{-/-} \text {MNCs (6) } \\
\text { AT1 } a^{-/-}+\text {WT-MNCs + VEGF mAb (5) }\end{array}$ & $\begin{array}{l}24.2 \pm 0.5 \\
24.9 \pm 0.5 \\
21.6 \pm 0.4 \\
22.7 \pm 0.3 \\
23.1 \pm 0.4 \\
23.2 \pm 0.6 \\
21.9 \pm 0.2 \\
22.3 \pm 0.3\end{array}$ & $\begin{array}{c}24.3 \pm 0.4 \\
25.2 \pm 0.6 \\
22.8 \pm 0.5 \\
23.1 \pm 0.5 \\
\text { NM } \\
\text { NM } \\
\text { NM } \\
\text { NM }\end{array}$ & $\begin{array}{l}24.1 \pm 0.3 \\
25.1 \pm 0.5 \\
22.2 \pm 0.4 \\
23.8 \pm 0.4 \\
23.4 \pm 0.4 \\
23.9 \pm 0.8 \\
23.1 \pm 0.2 \\
24.6 \pm 0.5\end{array}$ & $\begin{array}{l}24.7 \pm 0.4 \\
25.6 \pm 0.6 \\
24.0 \pm 0.4 \\
24.7 \pm 0.4 \\
24.5 \pm 0.2 \\
26.0 \pm 0.7 \\
24.4 \pm 0.3 \\
24.4 \pm 0.4\end{array}$ & $\begin{array}{l}24.5 \pm 0.4 \\
25.2 \pm 0.5 \\
24.6 \pm 0.5 \\
25.5 \pm 0.5 \\
25.6 \pm 0.2 \\
26.6 \pm 0.6 \\
25.6 \pm 0.2 \\
25.2 \pm 0.2\end{array}$ & $\begin{array}{l}24.9 \pm 0.4 \\
25.4 \pm 0.5 \\
25.5 \pm 0.5 \\
25.2 \pm 0.6 \\
25.9 \pm 0.2 \\
26.8 \pm 0.6 \\
25.8 \pm 0.2 \\
25.9 \pm 0.3\end{array}$ & $\begin{array}{l}25.7 \pm 0.4 \\
26.0 \pm 0.4 \\
25.5 \pm 0.5 \\
25.8 \pm 0.5 \\
25.9 \pm 0.2 \\
27.2 \pm 0.6 \\
26.3 \pm 0.1 \\
26.3 \pm 0.1\end{array}$ \\
\hline
\end{tabular}

VEGF mAb, neutralizing anti-VEGF mAb. NM, not measured. ${ }^{A} P<0.01$ vs. WT mice. SBP did not differ among the groups of $A T 1 a^{-/-}$, WT + TCV-116, WT + Hyd $A T 1 a^{/-}+$PD123319, AT1 $a^{-1-}+$ WT-MNCs, AT1 $a^{-/-}+A T 1 a^{-/-}$MNCs, and AT1 $a^{-/-}+$WT-MNCs + VEGF mAb. Body weight did not differ among all the groups.

Serial $L D B F$ analysis. Figure 1a shows representative LDBF images of hindlimb blood flow. Serial LDBF examination disclosed progressive recovery of hindlimb blood flow in WT mice after induction of ischemia (Figure 1, a and b). In contrast, the LDBF ratio of $A T 1 a^{-/}$mice remained impaired during the follow-up period, and the ratio of ischemic/normal LDBF was persistently low compared with WT mice $(P<0.05)$ (Figure 1b).

Angiographic score. We next examined whether the reduced $L D B F$ ratio was associated with the number of angiographically visible collateral vessels (arteriogenesis). On day 14, postmortem angiography revealed a lower number of collateral vessels in the thigh adductor muscle area of $A T 1 a^{-/-}$mice compared with WT mice (Figure 1c). Quantitative analysis of collateral vessels revealed that the angiographic score was significantly lower in $A T 1 a^{-/-}$mice than in WT mice (Figure 1d).

Tissue capillary density. To investigate the extent of angiogenesis at the microcirculation level, we measured capillary density in histologic sections harvested from the ischemic tissues. Figure 1e shows representative photomicrographs of tissue immunostained with CD31. Quantitative analysis revealed that on postoperative day 14 the capillary density was significantly reduced in $A T 1 a^{-/-}$mice compared with WT mice (Figure 1f).

Effects of hydralazine on angiogenesis in WT mice. SBP was lower in $A T 1 a^{-/-}$mice than in WT mice $(16,17)$. We therefore examined the effects of the lower SBP itself on ischemia-induced angiogenesis. We administered hydralazine to WT mice to lower their SBP to a level similar to that of $A T 1 a^{-/-}$mice (Table 1 ). The recovery of the LDBF ratio in hydralazine-treated WT mice after ischemia was completely preserved compared with nontreated WT mice (Figure 2). Thus, the reduced SBP itself did not impair angiogenesis in this mouse model. Effects of TCV-116 on angiogenesis in WT mice. We examined whether a pharmacological blockade of the AT1 receptor (by TCV-116) in WT mice would mimic the reduced angiogenic response observed in $A T 1 a^{-/-}$mice. WT mice $(n=15)$ were treated with TCV-116, a selective AT1 receptor antagonist. TCV-116 reduced the SBP of WT mice to a level similar to that of $A T 1 a^{-/-}$ mice (Table 1). TCV-116 impaired ischemia-induced angiogenesis in WT mice as assessed by the LDBF ratio, which was similar to the LDBF values observed in $A T 1 a^{-/-}$mice (Figure 2).

Effects of PD123319 on angiogenesis in AT1 $a^{-/-}$mice. In $A T 1 a^{-/-}$mice, the ATII-AT2 receptor pathway is still functioning and may potentially affect angiogenesis. Accordingly, we tested the effects of the selective AT2 receptor antagonist PD123319 on ischemia-induced angiogenesis in $A T 1 a^{-/-}$mice. PD123319 did not alter the SBP of $A T 1 a^{-/-}$mice $(n=5)$ (Table 1). After induction of limb ischemia, PD123319 did not influence the extent of angiogenesis in $A T 1 a^{-/-}$mice, as assessed by the ischemic/normal limb LDBF ratio (Figure 2).

Tissue inflammatory responses. The RAS plays proinflammatory roles $(18,25)$, and inflammation is an early trigger for ischemia-induced angiogenesis (31). We therefore examined histology of ischemic tissues on postoperative days 3 and 7. H\&E staining and CD45 immunostaining revealed a marked infiltration of inflammatory leukocytes in WT mice. In contrast, the number of infiltrated leukocytes was lower in the ischemic tissues of $A T 1 a^{-/-}$mice (Figure 3a). Moreover, the number of CD45-positive leukocytes was lower in $A T 1 a^{-/-}$mice than in WT mice (Figure $3 \mathrm{~b}$ ).

To further determine the type of infiltrated leukocytes, we stained tissue sections with F4/80 mAb (for macrophages), anti-CD3 mAb (for T lymphocytes), and anti-CD45 $\mathrm{mAb}$ (for common leukocyte antigen). The quantitative analysis revealed that the number of infiltrated macrophages and $\mathrm{T}$ lymphocytes was again lower in $A T 1 a^{-/-}$mice than in WT mice on days 3 and 7 (Figure 3d). 


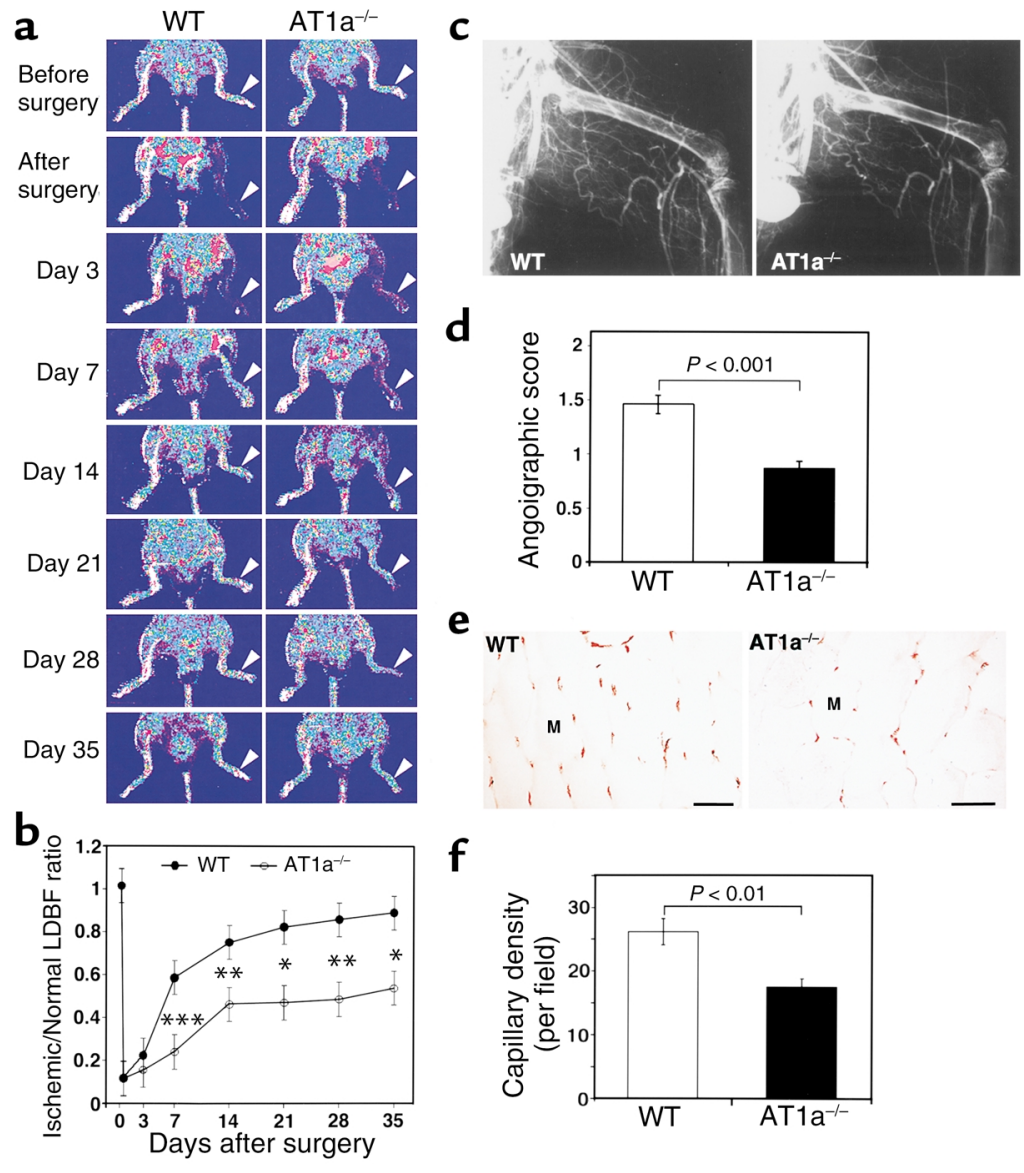

\section{Figure 1}

Angiogenesis was impaired in the ischemic hindlimb of $A T 1 a^{-/-}$mice. (a) A low perfusion signal (dark blue) was observed in the ischemic hindlimb of an $A T 1 a^{-/-}$mouse, whereas a high perfusion pattern (red to orange) was detected in a control WT mouse. (b) Computer-assisted quantitative analyses of hindlimb blood perfusion demonstrated a significant reduction in the ischemic/normal hindlimb blood flow ratio in AT1 $a^{-/-}$mice $(n=11)$ compared with WT animals $(n=13) .{ }^{*} P<0.05,{ }^{*} P<0.01,{ }^{*}{ }^{*} P<0.001$, WT vs. $A 1 a^{-1-}$. (c) Postmortem angiograms showed markedly reduced collateral vessel formation in AT1 $a^{-/-}$mouse compared with WT mouse. (d) Quantitative analysis revealed a low angiographic score in $A T 1 a^{-/-}$mice examined on postoperative day 14 ( $n=5$ in each group). (e) Immunostaining of ischemic tissues with anti-CD31 mAb (brown) showed markedly reduced capillary density in an $\mathrm{AT}_{1} \mathrm{a}^{-/-}$mouse compared with a WT mouse. (f) Quantitative analysis revealed a reduced capillary density in $A T 1 a^{-/-}$mice examined on postoperative day 14 ( $n=5$ in each group).

Inflammatory leukocytes express angiogenic cytokine VEGF. Inflammatory leukocytes release angiogenic cytokines including VEGF, which promote neovascularization (19, 28). To examine the relationship between leukocytes and VEGF expression, we performed double immunofluorescence staining for VEGF and leukocyte markers (CD3, F4/80, and CD45). There were VEGF- and F4/80-double positive macrophages as well as VEGFand CD3-double positive Tlymphocytes in ischemic tissues (Figure 3c). The number of VEGF-positive macrophages and $\mathrm{T}$ lymphocytes was lower in AT1 $\mathrm{a}^{-/-}$ mice than in WT mice on days 3 and 7 (Figure $3 \mathrm{~d}$ ). Double immunofluorescence staining showed that both macrophages and $\mathrm{T}$ lymphocytes were the major MNCs expressing VEGF. Skeletal myocytes in the ischemic tis-

\section{Figure 2}

Effects of hydralazine and ATII receptor blockers on ischemiainduced angiogenesis. No impairment in ischemic/normal hindlimb LDBF ratio was observed in WT mice treated with hydralazine (WT + Hydralazine) $(P=\mathrm{NS}$ vs. WT). Administration of the AT1 receptor blocker TCV-116 $(5 \mathrm{mg} / \mathrm{kg} / \mathrm{d})$ impaired ischemia-induced angiogenesis in WT mice (WT + TCV-116) $(P<0.01$ vs. WT $)$, so that the LDBF values were comparable to those observed in $A T 1 a^{-/-}$mice. Administration of the AT2 receptor blocker PD123319 (30 mg/kg/d) did not alter ischemiainduced angiogenesis in AT1 $a^{-/-}$mice (AT1 $a^{-/-}+$PD123319) $\left(P=\mathrm{NS}\right.$ vs. $\left.A T 1 a^{-/-}\right)$. sues also expressed VEGF, but the extent of VEGF expression was comparable in WT mice and $A T 1 a^{-/-}$ mice. There were only a few VEGF- and CD45-double positive polymorphonuclear leukocytes (PMNs) (i.e., negative for both F4/80 and CD3) in ischemic tissues.

Because VEGF and MCP-1 are the major cytokines responsible for ischemia-induced angiogenesis as well as arteriogenesis $(28-30,32,33)$, and because MCP-1 can recruit monocytes, we performed Western blot analysis to examine the endogenous expression of VEGF and MCP-1 in the ischemic tissues of WT mice and $A T 1 a^{-/-}$mice. Western blot analysis revealed a marked reduction in the expression of both VEGF and MCP-1 proteins in AT1 $a^{-/-}$mice compared with WT mice on postoperative days 3 and 7 (Figure 3e).

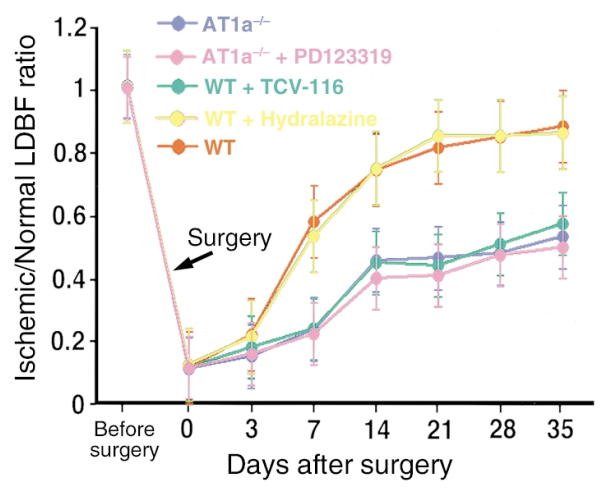




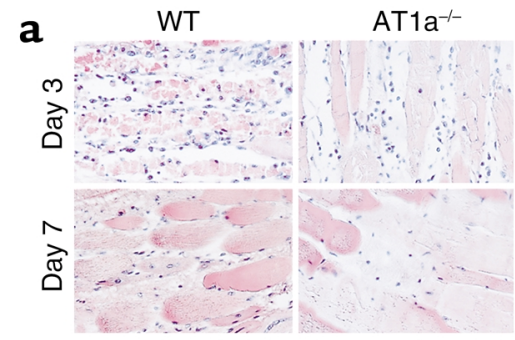

C

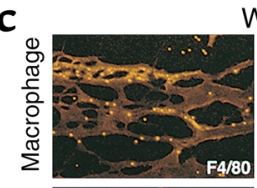

WT
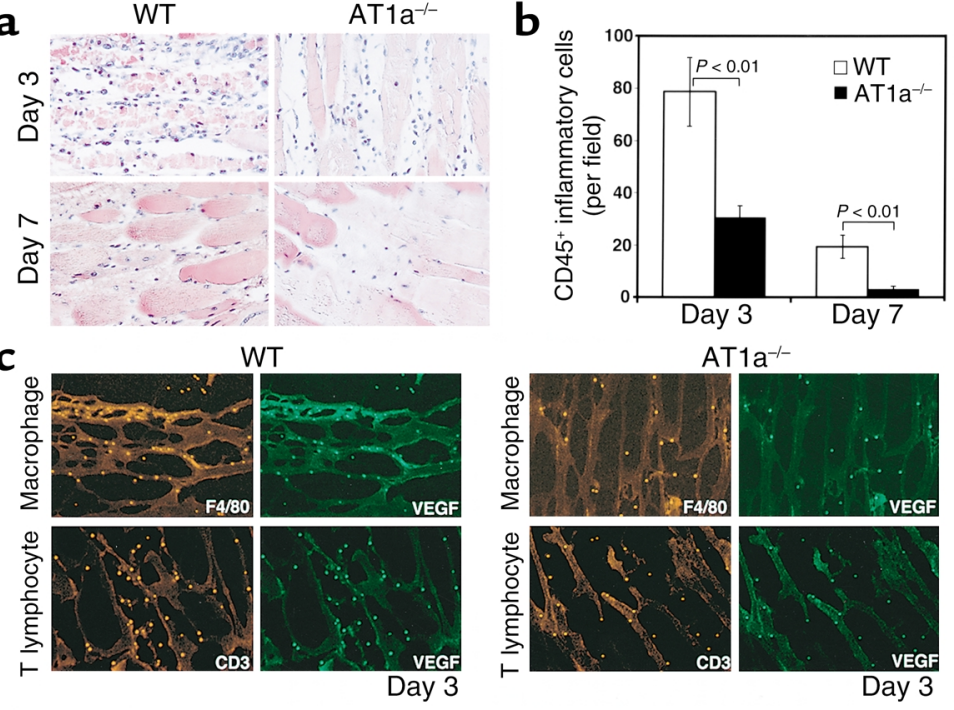

AT1 $\mathrm{a}^{-1-}$

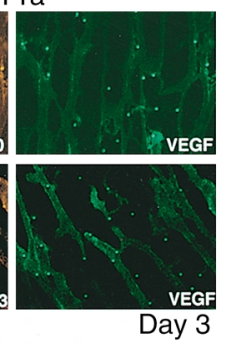

d
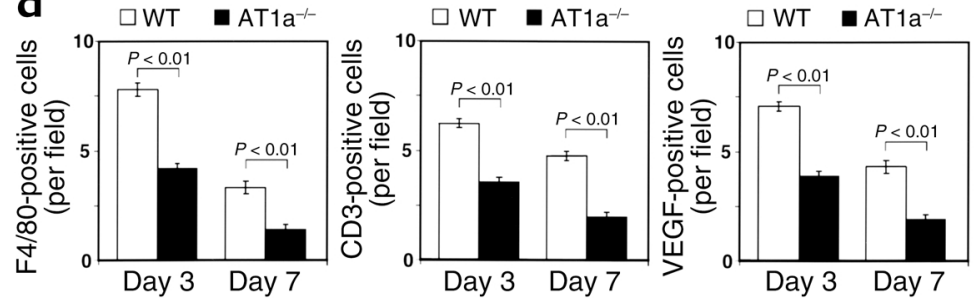

e

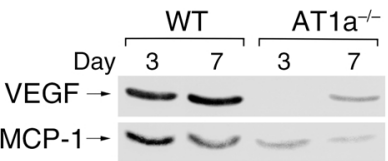

In vivo leukocyte migration assay using a peritonitis model. We examined whether leukocyte infiltration was reduced in another model of inflammatory response in $A T 1 a^{-/-}$mice. Intraperitoneal administration of oyster glycogen induces peritonitis, which is an established inflammation model in mice (29). In this model, peritonitis was associated with a marked accumulation of inflammatory leukocytes in the peritoneal cavity of WT mice $\left(1.8 \times 10^{6} \pm 0.2 \times 10^{6}\right.$ cells, $n=10)$. However, in $A T 1 a^{-/-}$mice $(n=10)$, there were only $1.1 \times 10^{6} \pm 0.1 \times 10^{6}$ cells $(P<0.01$ vs. WT mice) . Thus, the AT1a receptor deficiency was associated with reduced transendothelial migration of leukocytes in the peritonitis model.

Effects of the implantation of WT mouse- or AT1 $a^{-/-}$ mouse-derived peripheral blood MNCs on angiogenesis in $A T 1 a^{-/-}$mice. Because ischemic tissues of $A T 1 a^{-/-}$mice contained fewer inflammatory leukocytes with VEGF expression compared with WT mice, we examined whether impaired angiogenesis in $A T 1 a^{-/-}$mice could be rescued by implantation of WT mouse- or AT1 $a^{-/-}$ mouse-derived peripheral blood MNCs. LDBF analysis showed that implantation of WT-derived MNCs into the ischemic hindlimb significantly restored the ischemic/normal LDBF ratio in $A T 1 a^{-/-}$mice compared with nontransplanted $A T 1 a^{-/-}$mice (Figure 4). In contrast, implantation of $A T 1 a^{-/-}$mouse-derived MNCs

\section{Figure 3}

Inflammatory responses and expression of angiogenic cytokines in ischemic tissues. (a) H\&E staining revealed marked infiltration of inflammatory leukocytes in the ischemic tissues of WT mice but not in those of $A T 1 a^{-/-}$ mice. (b) Quantitative analysis revealed reduced inflammatory cell infiltration in $A T 1 a^{-/-}$mice examined on days 3 and 7 after induction of limb ischemia $(n=4$ in each group). (c) Double immunofluorescence staining of the ischemic tissues revealed that macrophages and T lymphocytes expressed VEGF protein. VEGF showed green fluorescence, whereas cell surface markers (F4/80 and CD3) were stained in red. Skeletal myocytes in the ischemic tissues also moderately expressed VEGF protein. (d) Quantitative analysis revealed that the number of infiltrating macrophages and T lymphocytes was significantly smaller in AT1 $a^{-1-}$ mice than in WT mice. The number of VEGF-positive inflammatory cells was also significantly lower in $A T 1 a^{-/-}$mice than in WT mice. (e) Western blot analysis revealed that the expression of VEGF and MCP-1 proteins in the ischemic tissues was weaker in $A T 1 a^{-/-}$mice than in WT mice on days 3 and 7 after induction of limb ischemia.

into the ischemic limb restored the ischemic/normal LDBF ratio slightly in $A T 1 a^{-/-}$mice, but the values were not significantly different from those of $A T 1 a^{-/-}$ mice (Figure 4). Finally, under the treatment with a neutralizing anti-VEGF $\mathrm{mAb}$, implantation of WT mouse-derived MNCs into the ischemic hindlimb no longer restored the angiogenesis in $A T 1 a^{-/-}$mice, suggesting an important role of VEGF produced by MNCs in the rescue of ischemia-induced angiogenesis.

\section{Discussion}

Angiogenesis, arteriogenesis, and blood flow recovery in response to hindlimb ischemia were significantly impaired in $A T 1 a^{-/-}$compared with WT mice. Moreover, pharmacological blockade of the AT1 receptor by TCV-116 suppressed the ischemia-induced angiogenesis in WT mice. The impaired angiogenesis was not due to the reduced SBP, since hydralazine treatment did not influence angiogenesis. Therefore, the present study, for the first time to our knowledge, provides in vivo evidence that the ATII-AT1 receptor pathway is important for ischemia-induced angiogenesis. Our findings further extend previous reports indicating that ATII functions as a proangiogenic molecule (32-34). For instance, ATII induces VEGF expression in vascular smooth muscle cells, which stimulates EC proliferation, migration, and angiogenesis $(32,33)$. ATII also enhances VEGF-mediated angiogenesis (35).

ATII functions through AT1a and AT2 receptors in mice, and the RAS is activated in $A T 1 a^{-/-}$mice $(16,17)$. Therefore, in $A T 1 a^{-/-}$mice, the ATII-AT2 receptor pathway is still functioning and could affect angiogenesis. Therefore, we tested the effects of the selective AT2 
receptor antagonist PD123319 on ischemia-induced angiogenesis in $A T 1 a^{-/-}$mice. PD123319 did not influence the extent of angiogenesis in $A T 1 a^{-/}$mice as assessed by the LDBF ratio, indicating that the ATII-AT2 receptor pathway may play a minimum role in modulating ischemia-induced angiogenesis in $A T 1 a^{-/-}$mice. Our results are consistent with a previous report showing that the expression of AT2 receptor was low in vascular tissues and thus the ATII functioned through AT2 receptor only weakly within skeletal muscles (36).

We next sought to explore the potential mechanism(s) for the impaired angiogenesis in $A T 11^{-/-}$mice. During the examination of tissue histology of the ischemic skeletal muscles, we found a marked infiltration of inflammatory leukocytes in WT mice, whereas the number of leukocytes was lower in $A T 1 a^{-/-}$mice. Moreover, the number of infiltrated macrophages and $\mathrm{T}$ lymphocytes was significantly lower in $A T 1 a^{-/}$mice than in WT mice on days 3 and 7. In the present study, transendothelial migration of leukocytes was also suppressed in $A T 1 a^{-/-}$mice in the glycogen-induced peritonitis model. Our findings are consistent with previous studies indicating that ATII supported leukocyte transendothelial migration via an AT1 receptor-dependent, but blood pressure-independent, mechanism (37-39). Because inflammation is an early key process for ischemia-induced angiogenesis and arteriogenesis (31), it is conceivable that the deficiency of the AT1a receptor may have resulted in reduced inflammatory reactions, and that, consequently, angiogenesis was impaired. In this regard, recent studies showed that the RAS exists not only in the vascular wall but also in immune-privileged tissues and cells $(26,27)$, which play important proinflammatory roles $(18,25)$.

Infiltrated inflammatory leukocytes release angiogenic cytokines including VEGF, which promote neovascularization $(19,28)$. In fact, previous studies showed that the infiltration of VEGF-positive $\mathrm{T}$ lymphocytes and macrophages plays an important role in ischemiainduced and tumor-related angiogenesis $(19,28,40,41)$. In the present study, infiltrated macrophages and $T$ lymphocytes expressed VEGF intensively, and the number of VEGF-positive macrophages and $T$ lymphocytes was lower in $A T 1 a^{-/}$mice than in WT mice. There were only a few VEGF- and CD45-double positive PMNs lacking F4/80 and CD3 in ischemic tissues. The latter result was consistent with a report by Koehne et al. (42), who showed a lack of hypoxic stimulation of VEGF expression in PMNs. Skeletal myocytes in the ischemic tissues occasionally expressed moderate amounts of VEGF, but the extent of VEGF expression was almost comparable between WT mice and $A T 1 a^{-/-}$mice. Taken together, infiltrated MNCs (i.e., macrophages and $\mathrm{T}$ lymphocytes) released VEGF protein, which might have facilitated ischemia-induced angiogenesis.

In the present study, arteriogenesis (i.e., angiographically visible collateral vessel formation) was also impaired in $A T 1 a^{-/-}$mice compared with WT mice. Arte- riogenesis is mainly mediated by the chemokine MCP-1 $(43,44)$, which is a key molecule for the recruitment of monocytes and macrophages. Recent studies documented that not only angiogenesis but also arteriogenesis in response to tissue ischemia depended on macrophage infiltration $(31,40,41,43)$. We therefore examined the tissue contents of both VEGF and MCP-1 in the ischemic hindlimb tissues of WT mice and $A T 1 a^{-1-}$ mice. Western blot analysis revealed a marked reduction in the expression of both VEGF and MCP-1 proteins in $A T 1 a^{-/}$mice compared with WT mice on postoperative days 3 and 7 . This indicates that reduced macrophage infiltration and repression of angiogenic cytokines, such as VEGF and MCP-1, may account at least in part for the reduced macrophage infiltration as well as suppressed arteriogenesis in $A T 1 a^{-1-}$ mice.

To further determine the functional role of the infiltration of MNCs in angiogenesis, we tested whether implantation of WT mouse-derived MNCs or $A T 1 a^{-/}$ mouse-derived MNCs into the ischemic tissues could rescue the impaired angiogenesis in $A T 1 a^{-/-}$mice. We previously showed that direct tissue implantation of bone marrow MNCs or human cord blood-derived endothelial precursor cells augmented neovascularization in vivo $(45,46)$. LDBF analysis showed that implantation of WT mouse-derived MNCs into the ischemic limb significantly restored the ischemic/normal LDBF ratio in $A T 1 a^{-/}$mice. In contrast, implantation of $A T 1 a^{-/-}$ mouse-derived MNCs into the ischemic limb slightly restored the LDBF ratio in $A T 1 a^{-/-}$mice, but the values were not significantly different from those of $A T 1 a^{-/-}$ mice. These findings suggest that inflammatory MNC infiltration is indeed an important event for the ischemiainduced angiogenesis. Moreover, the results suggest that the expression of AT1a receptor on MNCs is also important for inflammatory cell-mediated angiogenesis.

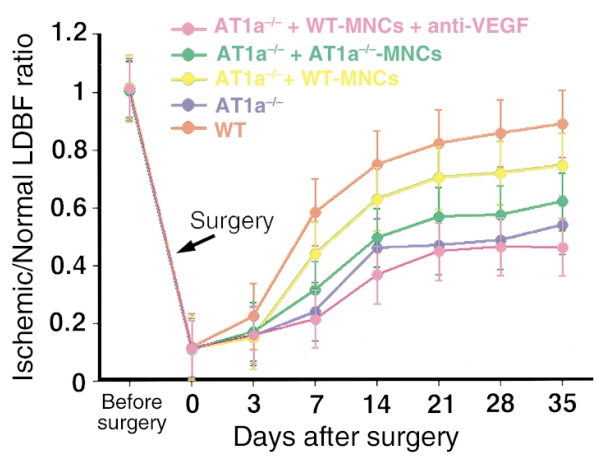

\section{Figure 4}

Impacts of the implantation of WT mouse- or AT1 $a^{-/-}$mouse-derived MNCs into the ischemic hindlimbs of $A T 1 a^{-/-}$mice on angiogenesis. The impaired LDBF ratio in $A T 1 a^{-/-}$mice was partially but significantly restored after implantation of WT mouse-derived MNCs into the ischemic hindlimb of $A T 1 a^{-/-}$mice $\left(P<0.01 \mathrm{vs}\right.$. AT1 $\left.a^{-/-}\right)$. In contrast, implantation of $A T 1 a^{-/-}$mouse-derived MNCs slightly improved the LDBF ratio in $A T 1 a^{-/-}$mice, but the difference was not significant (vs. $A T 1 a^{-/-}$). Neutralizing anti-VEGF mAb treatment abolished the WT-MNC-mediated rescue of impaired angiogenesis in AT $1 a^{-/-}$mice $\left(P=\right.$ NS vs. $\left.A T 1 a^{-/-}\right)$. 
Finally, we examined whether VEGF released from transplanted WT-derived MNCs played a role in the rescue of angiogenesis in $A T 1 a^{-/-}$mice. We tested the effects of a neutralizing anti-VEGF $\mathrm{mAb}$ on angiogenesis in $A T 1 a^{-/}$mice subjected to WT-derived MNC implantation. The anti-VEGF mAb treatment attenuated the WT-MNC-mediated rescue of the impaired angiogenesis in $A T 1 a^{-/-}$mice. This result further indicates that the implantation of WT-derived MNCs rescued the angiogenesis in $A T 1 a^{-/-}$mice mainly via a VEGF-dependent pathway.

There are several limitations to our study. First, the dose of TCV-116 was higher than the clinical dose (47). However, this dose was necessary to reduce the SBP of WT mice to a level similar to that of $A T 1 a^{-/-}$mice, and this dose has been commonly used in animal models to block the AT1 receptor in vivo (21-23). Second, in several experimental groups, we measured only LDBF values to assess ischemia-induced angiogenesis. However, we and others previously demonstrated that tissue capillary density was well correlated with the ischemic/normal LDBF ratio in this mouse model of angiogenesis $(10,19)$. Thus, we believe that the LDBF ratio represents the extent of angiogenesis in the ischemic hindlimb. Third, Western blot analysis demonstrated lower expression of VEGF protein in homogenized tissue extracts in $\mathrm{AT}^{-a^{-/-}}$mice than in WT mice. This method does not address the possibility that the expression of VEGF in skeletal muscles may differ between WT and AT1 $a^{-/-}$mice. However, this possibility is less likely, since our immunohistochemical staining revealed a comparable expression of VEGF in skeletal myocytes. Moreover, skeletal myocytes express only low amounts of AT1 and AT2 receptors $(36,48)$, and thus skeletal muscle VEGF expression may be little influenced by ATII.

In summary, our findings suggest that the ATII-AT1 receptor pathway plays an important role in ischemiainduced angiogenesis in vivo. The mechanism is likely related to the ATII-mediated inflammatory MNC infiltration. These are newly discovered functions of the ATII-AT1 receptor pathway in vivo. Moreover, our results have several clinical implications. First, there may be a therapeutic window for AT1 receptor antagonists in patients with acute ischemic diseases. Because angiogenesis is an important survival mechanism for tissues exposed to ischemia (49), the use of an AT1 receptor blocker early after ischemia may suppress subsequent angiogenesis, resulting in exacerbation of ischemia. Second, the suppression of inflammatory cell infiltration by the AT1 receptor blockage would provide a unique strategy against angiogenic disorders, including malignant tumors. In fact, recent studies suggest that infiltration of macrophages and $\mathrm{T}$ lymphocytes promote tumor-related angiogenesis $(28,50)$.

\section{Acknowledgments}

This study was supported by research grants $(11158220,12032220$, and 12470161) and a Scientific
Frontier grant from the Ministry of Education, Science, and Culture, the Tokyo Biochemical Research Society, the Japan Heart Foundation, and grants from the Ministry of Health and Welfare (to T. Murohara). We thank K. Kimura, K. Moriyama, and M. Aoki for technical assistance, Takeda Chemical Industries for TCV-116, and Novartis Pharma for hydralazine.

1. Brunner, H.R., et al. 1972. Essential hypertension: rennin and aldosterone, heart attack and stroke. N. Engl. J. Med. 286:441-449.

2. Lever, A.F., et al. 1998. Do inhibitors of angiotensin-I-converting enzyme protect against risk of cancer? Lancet. 352:179-184.

3. Folkman, J. 1995. Angiogenesis in cancer, vascular, rheumatoid and other disease. Nat. Med. 1:27-31.

4. Hanahan, D., and Folkman, J. 1996. Patterns and emerging mechanisms of the angiogenic switch during tumorigenesis. Cell. 86:353-364.

5. Chaturvedi, N., et al. 1998. Effect of lisinopril on progression of retinopathy in normotensive people with type 1 diabetes. The EUCLID Study Group. EURODIAB Controlled Trial of Lisinopril in InsulinDependent Diabetes Mellitus. Lancet. 351:28-31.

6. Volpert, O.V., et al. 1996. Captopril inhibits angiogenesis and slows the growth of experimental tumors in rats. J. Clin. Invest. 98:671-679.

7. Fabre, J.-E., Rivard, A., Magner, M., Silver, M., and Isner, J.M. 1999. Tissue inhibition of angiotensin-converting enzyme activity stimulates angiogenesis in vivo. Circulation. 99:3043-3049.

8. Warren, J.B., and Loi, R.K. 1995. Captopril increases skin microvascular blood flow secondary to bradykinin, nitric oxide, and prostaglandins. FASEB J. 9:411-418

9. Emanueli, C., et al. 2001. Local delivery of human tissue kallikrein gene accelerates spontaneous angiogenesis in mouse model of hindlimb ischemia. Circulation. 103:125-132.

10. Murohara, T., et al. 1998. Nitric oxide synthase modulates angiogenesis in response to tissue ischemia. J. Clin. Invest. 101:2567-2578.

11. Muramatsu, M., Katada, J., Hayashi, I., and Majima, M. 2000. Chymase as a proangiogenic factor. A possible involvement of chymaseangiotensin-dependent pathway in the hamster sponge angiogenesis model. J. Biol. Chem. 275:5545-5552.

12. Timmermans, P.B., et al. 1993. Angiotensin II receptors and angiotensin II receptor antagonists. Pharmacol. Rev. 45:205-251.

13. Burnier, M. 2001. Angiotensin II type 1 receptor blockers. Circulation. 103:904-912.

14. Viswanathan, M., Stromberg, C., Seltzer, A., and Saavedra, J.M. 1992. Balloon angioplasty enhances the expression of angiotensin II AT1 receptors in neointima of rat aorta. J. Clin. Invest. 90:1707-1712.

15. Harada, K., Komuro, I., Sugaya, T., Murakami, K., and Yazaki, Y. 1999 Vascular injury causes neointimal formation in angiotensin II type 1a receptor knockout mice. Circ. Res. 84:179-185.

16. Sugaya, T., et al. 1995. Angiotensin II type 1a receptor-deficient mice with hypotension and hyperreninemia. J. Biol. Chem. 270:18719-18722.

17. Ito, M., et al. 1995. Regulation of blood pressure by the type $1 \mathrm{~A}$ angiotensin II receptor gene. Proc. Natl. Acad. Sci. USA. 92:3521-3525.

18. Hisada, Y., et al. 1999. Angiotensin II plays a pathogenic role in immunemediated renal injury in mice. J. Clin. Invest. 103:627-635.

19. Couffinhal, T., et al. 1998. A mouse model of angiogenesis. Am. J. Pathol. 152:1667-1679.

20. Duan, J., et al. 2000. Hypercholesterolemia inhibits angiogenesis in response to hindlimb ischemia: nitric oxide-dependent mechanism. Circulation. 102(Suppl. III):III-370-III-376.

21. Yoshiyama, M., et al. 1994. Cardioprotective effect of the angiotensin II type 1 receptor antagonist TCV-116 on ischemia-reperfusion injury. Am. Heart J. 128:1-6.

22. Kojima, M., et al. 1994. Angiotensin II receptor antagonist TCV-116 induces regression of hypertensive left ventricular hypertrophy in vivo and inhibits the intracellular signaling pathway of stretch-mediated cardiomyocyte hypertrophy in vitro. Circulation. 89:2204-2211.

23. Nio, Y., Matsubara, H., Murasawa, S., Kanasaki, M., and Inada, M. 1995. Regulation of gene transcription of angiotensin II receptor subtypes in myocardial infarction. J. Clin. Invest. 95:46-54.

24. Levy, B.I., et al. 1996. Chronic blockade of AT2-subtype receptors prevents the effect of angiotensin II on the rat vascular structure. J. Clin. Invest. 98:418-425.

25. Usui, M., et al. 2000. Important role of local angiotensin II activity mediated via type 1 receptor in the pathogenesis of cardiovascular inflammatory changes induced by chronic blockade of nitric oxide synthesis in rats. Circulation. 101:305-310.

26. Okamura, A., et al. 1999. Upregulation of renin-angiotensin system during differentiation of monocytes to macrophages. J. Hypertens. 17:537-545.

27. Nataraj, C., et al. 1999. Angiotensin II regulates cellular immune 
responses through a calcineurin-dependent pathway. J. Clin. Invest. 104:1693-1701.

28. Freeman, M.R., et al. 1995. Peripheral blood T lymphocytes and lymphocytes infiltrating human cancers express vascular endothelial growth factor: a potential role for $\mathrm{T}$ cells in angiogenesis. Cancer Res. 55:4140-4145

29. Vaporciyan, A.A., et al. 1993. Involvement of platelet-endothelial cell adhesion molecule-1 in neutrophil recruitment in vivo. Science. 262:1580-1582

30. Murohara, T., Delyani, J.A., Albelda, S.M., and Lefer, A.M. 1996. Blockade of platelet endothelial cell adhesion molecule- 1 protects against myocardial ischemia and reperfusion injury in cats. J. Immunol. 156:3550-3557.

31. Arras, M., et al. 1998. Monocyte activation in angiogenesis and collateral growth in the rabbit hindlimb. J. Clin. Invest. 101:40-50.

32. Williams, B., Baker, A.Q., Gallacher, B., and Lodwick, D. 1995 Angiotensin II increases vascular permeability factor gene expression by human vascular smooth muscle cells. Hypertension. 25:913-917.

33. Richard, D.E., Berra, E., and Pouyssegur, J. 2000. Nonhypoxic pathway mediates the induction of hypoxia-inducible factor 1alpha in vascular smooth muscle cells. J. Biol. Chem. 275:26765-26771.

34. Moravski, C.J., et al. 2000. Retinal neovascularization is prevented by blockade of the renin-angiotensin system. Hypertension. 36:1099-1104.

35. Otani, A., Takagi, H., Suzuma, K., and Honda, Y. 1998. Angiotensin II potentiates vascular endothelial growth factor-induced angiogenic activity in retinal microcapillary endothelial cells. Circ. Res. 82:619-628.

36. Malendowicz, S.L., et al. 2000. Angiotensin II receptor subtypes in the skeletal muscle vasculature of patients with severe congestive heart failure. Circulation. 102:2210-2213.

37. Mervaala, E.M.A., et al. 1999. Monocyte infiltration and adhesion molecules in a rat model of high human renin hypertension. Hypertension. 33:389-395.

38. Strawn, W.B., Gallagher, P.E., Tallant, E.A., Ganten, D., and Ferrario, C.M. 1999. Angiotensin II AT1-receptor blockade inhibits monocyte activation and adherence in transgenic (mRen2)27 rats. J. Cardiovasc. Pharmacol. 33:341-351.

39. Pastore, L., et al. 1999. Angiotensin II stimulates intercellular adhesion molecule-1 (ICAM-1) expression by human vascular endothelial cells and increases soluble ICAM-1 release in vivo. Circulation. 100:1646-1652.

40. Couffinhal, T., et al. 1999. Impaired collateral vessel development associated with reduced expression of vascular endothelial growth factor in apoE-/- mice. Circulation. 99:3188-3198.

41. Rivard, A., et al. 1999. Age-dependent impairment of angiogenesis. Circulation. 99:111-120.

42. Koehne, P., et al. 2000. Lack of hypoxic stimulation of VEGF secretion from neutrophils and platelets. Am. J. Physiol. 279:H817-H824.

43. Ito, W.D., et al. 1997. Monocyte chemotactic protein-1 increases collateral and peripheral conductance after femoral artery occlusion. Circ. Res. 80:829-837.

44. Hoefer, I.E., van Royen, N., Buschmann, I.R., Piek, J.J., and Schaper, W. 2001. Time course of arteriogenesis following femoral artery occlusion in the rabbit. Cardiovasc. Res. 49:609-617.

45. Murohara, T., et al. 2000. Transplanted cord blood-derived endothelial precursor cells augment postnatal neovascularization. J. Clin. Invest. 105:1527-1536

46. Shintani, S., et al. 2001. Augmentation of postnatal neovascularization with autologous bone marrow transplantation. Circulation. 103:897-903.

47. Delacretaz, E., Nussberger, J., Biollaz, J., Waeber, B., and Brunner, H.R. 1995. Characterization of the angiotensin II receptor antagonist TCV116 in healthy volunteers. Hypertension. 25:14-21.

48. de Gasparo, M., et al. 1995. Proposed update of angiotensin receptor nomenclature. Hypertension. 25:924-927.

49. Isner, J.M., and Asahara, T. 1999. Angiogenesis and vasculogenesis as therapeutic strategies for postnatal neovascularization. J. Clin. Invest. 103:1231-1236.

50. Leek, R.D., et al. 1996. Association of macrophage infiltration with angiogenesis and prognosis in invasive breast carcinoma. Cancer Res. 56:4625-4629. 